\title{
Transatlantica
}

Revue d'études américaines. American Studies Journal

\section{Les modalités du temps chez Benjamin Franklin}

\section{Elise Marienstras}

\section{(2) OpenEdition}

\section{Journals}

\section{Édition électronique}

URL : https://journals.openedition.org/transatlantica/4567

DOI : $10.4000 /$ transatlantica.4567

ISSN : 1765-2766

\section{Éditeur}

Association française d'Etudes Américaines (AFEA)

\section{Référence électronique}

Elise Marienstras, «Les modalités du temps chez Benjamin Franklin», Transatlantica [En ligne], 2 | 2009, mis en ligne le 11 janvier 2010, consulté le 01 février 2023. URL : http://

journals.openedition.org/transatlantica/4567 ; DOI : https://doi.org/10.4000/transatlantica.4567

Ce document a été généré automatiquement le 1 février 2023.

\section{(c) (1) (9)}

Creative Commons - Attribution - Pas d'Utilisation Commerciale - Pas de Modification 4.0 International - CC BY-NC-ND 4.0

https://creativecommons.org/licenses/by-nc-nd/4.0/ 


\title{
Les modalités du temps chez Benjamin Franklin
}

\author{
Elise Marienstras
}

Tout passe, tout se passe. [...] Cela se passe ; mais « cela » n'existe pas en soi, n'est pas chose en soi, n'est pas en dehors du fait de passer. Il n'y a pas de « chose » dans le temps phénoménal. [...] C'est le temps sans substance, le temps dont l'analogie la plus parfaite se retrouverait dans la musique. Bernard Groethuysen, Philosophie et histoire

1 Parler du temps, de ou chez Benjamin Franklin, peut paraître sans objet. Ce pourrait être aussi bien embrasser sa vie, ses contemporains, la société qui l'abrita, que son imaginaire, sa réflexion, sa volonté, ou encore son action sur lui-même et sur l'espace qui l'entourait. Et pourtant, ce concept de «temps » peut se révéler l'un des meilleurs outils pour percer l'énigme de ce personnage exceptionnel, mais si complexe que fut Franklin.

2 Mais le temps: quel mot banal ! Quel objet, quel concept est-il plus commun aux hommes, à la vie même, jusque - et y compris - à la pensée de la mort ? Quelle expérience est-elle plus quotidienne, plus variée, plus remplie de toute la gamme des sentiments, des émotions qui sont le lot de tous les êtres animés ? Quel mot est-il plus polysémique, plus présent dans toutes disciplines, plus porteur d'heuristique? Dans la langue française, combinant les deux sens de l'anglais «time» et " weather ", le mot « temps » évoque, dans la question « quel temps fait-il? », les phénomènes naturels les plus prosaïques.

3 Le temps est la dimension qui, plus encore que l'espace, commande tout ce qui concerne l'individu et la société : c'est un facteur abstrait, qui peut s'inscrire dans l'endeçà et dans l'au-delà, à la fois pragmatique, immanent et métaphysique ou transcendant. En Occident, le temps est au fondement des cultures nées des héritages antique, biblique et helléniste, romain et impérial, féodal et chrétien. Sa conception s'est modelée successivement sur une vision du monde providentialiste, et sur la 
sécularisation rationnelle, dite "éclairée » des cultures, promue par les philosophes européens des Lumières et leurs correspondants des Amériques. Le concept de temps est présent, en effet, dans l'interrogation de tous les philosophes européens du XVIe au XVIIIe siècle. Jusqu'alors, il s'agissait de «faire la part du temps », c'est-à-dire, comme l'écrit Guillaume Pigeart de Gurbert, de« faire le départ entre ce qui participe du temps et de ce qui n'en fait pas partie » (Pigeard de Guibert, Tunstall et Salem). « La véritable découverte $d u$ siècle des Lumières est qu'il n'est plus possible de faire la part du temps ", écrit encore cet auteur. Et il conclut : "Le temps des Lumières n'est plus un concept auquel on puisse assigner une place déterminée, c'est l'élément même dans lequel baigne le tout de l'être ».

4 Ajoutons que, des deux dimensions que nous prêtons à l'univers - le temps et l'espace -, le temps est la plus insaisissable; c'est celle dont on dit avec justesse qu'elle nous gouverne, celle sur laquelle nous imprimons le moins notre marque. Et pourtant, Benjamin Franklin se voulut maître du temps dont il fit l'instrument de sa volonté, de son ascension, de son bonheur ainsi que du progrès de l'humanité tout entière, comme le voulait l'esprit des Lumières dans lesquelles il baigna. Cette universalité même du concept rend la tâche difficile lorsqu'on se prépare à examiner les modalités du «temps" telles qu'elles s'expriment en particulier chez Benjamin Franklin. Les occurrences du mot «temps » dans ses écrits sont innombrables. Car le «temps » est, pour lui, un sujet des plus importants, tant sur la longue durée que dans la quotidienneté. Nous verrons qu'il perçoit le temps à la fois comme une opportunité pour l'individu d'exercer sa libre volonté dans la conduite de sa propre vie et comme un élément qui gouverne l'univers.

\section{Le maître du temps}

5 Dans l'almanach Poor Richard Improved, Franklin écrit en 1758: " doest thou love Life, then do not squander Time, for that's the stuff/Life is made of, as poor Richard says $»^{1}$. Ce penchant du philosophe à s'approprier son destin, à peser de toutes ses forces et de toute son intelligence sur le présent et l'avenir des hommes, à s'entremettre pour qu'entre passé, présent et futur, le temps des générations échappe à la discontinuité, Franklin le partage-t-il avec ses contemporains, ou n'y met-il pas surtout sa singularité, sa complexité, ses contradictions?

On réduit souvent la conception du «temps» des hommes des Lumières à sa concordance avec la notion de "progrès ", sorte de fuite en avant selon une ligne droite qui se dessine chez l'être humain comme dans ses réalisations. Franklin, comme nombre de ses concitoyens, partage largement cette vision. Intimement lié au concept de "temps ", celui de "progrès » est présent à tout moment de la pensée de Franklin, sans toutefois englober tous les modes de la temporalité dont use ce personnage souvent déroutant par sa versatilité, capable de puiser dans des traditions idéologiques aussi disparates que le sont les usages du mot «temps ». Surtout, il a une conscience aiguë de l'écoulement du temps, mais il se refuse à le subir.

Dans l'effort constant qu'il dit exercer à maîtriser le temps dont il dispose, à affiner son esprit, à atteindre à la plénitude de son être, il nourrit le temps qui passe de tout un assortiment de tâches et de plus ou moins plaisantes occupations; il croit pouvoir retenir le flux des minutes et des heures, les allonger ou les contracter de sorte à 
mettre la durée au service de la réussite de sa personne, de l'accomplissement de son être, de la place qu'il occupe dans la société de son époque.

Peu préoccupé par l'eschatologie ou d'autres finalités du temps - mortalité et postérité, éternité ${ }^{-}$, il inscrit plutôt sa vie, sa pensée, son action dans le temps présent. Il use de toute l'étendue des sens du mot " temps ", qui figure dans tous les champs de la pensée, de la science, et, comme ses contemporains, il fonde sa pratique existentielle sur le bon usage du temps, auquel le "Pauvre Richard» consacre une grande partie de ses maximes et aphorismes.

Paradoxalement, si rationnel que se veuille le «Bonhomme Franklin», son appréhension du temps participe aussi de la singularité des Lumières américaines, une singularité faite du sentiment de ces Européens d'origine que le «nouveau » continent dans lequel ils ont échu ne doit rien ou si peu à la «vieille » Europe. Dans l'affectation idéologique des retrouvailles avec les grands mythes antiques, le «temps» d'au-delà l'océan a fait machine arrière. Les grands Champs élyséens que représente la terre américaine pour l'imaginaire émerveillé des Européens des XVIe et XVIIe siècles les portent en esprit à l'aube bénie des temps antiques panthéistes ou dans un Eden ininterrompu épargné par le péché originel et la malédiction divine (Pagden, Marienstras 1976).

10 Cependant, il aurait été vain de vouloir recréer une civilisation de progrès dans la neuve Amérique si, pour créer une nation semblable aux autres, et même supérieure, les «pèlerins" puis les révolutionnaires avaient effacé tout passé et s'étaient totalement déplacés hors du temps historique. Les inventeurs de la nation américaine, loin de jouer les sauvages perdus dans la nature des premiers temps, se réclament des Lumières, au zénith de la civilisation européenne. Tout en participant au mouvement de la «table rase » révolutionnaire, John Adams, Thomas Jefferson, Benjamin Franklin, ces deux derniers empressés dans les salons parisiens, assidus dans leur correspondance avec les philosophes du Vieux monde, se gardent donc bien de renier le grand moment historique des Lumières et d'en dissocier la naissance de la nation américaine. C'est ainsi que le «moment » Franklin concorde avec le «moment » éclairé de l'humanité et avec le «moment » historique de l'Amérique par lequel les États-Unis naissent en révolution. John Adams et Benjamin Franklin relèvent à maintes reprises que cette nation a eu la chance de voir le jour à «l'ère de raison ».

11 C'est cette concordance des temps qui a fait de Franklin un révolutionnaire. Il fut, en un sens, représentatif de ce que fut cette révolution : s'il avait pu, lui qui ne dédaignait pas de fréquenter la noblesse anglaise ou française et qui n'était pas un foudre de guerre, échanger sans bruit la royauté pour la république par la négociation diplomatique dans laquelle il excellait, il aurait sans doute choisi de ne chercher la victoire que par sa plume et même de rompre avec l'Angleterre en se contentant de la quitter, comme il l'avait fait lorsque, à seize ans, il avait fui le carcan puritain de Nouvelle-Angleterre pour exercer librement sa pensée loin de la superstition et de l'obscurantisme (reproche qu'il fera injustement à Cotton Mather ${ }^{3}$ ) et grimper allègrement à l'échelle sociale sous des cieux plus tolérants.

\section{Franklin et le temps des Lumières}

12 Dans ce siècle américain de la raison qu'illustre si bien Franklin, les laudateurs de la nation nouvelle dessinent une double temporalité pour légitimer la rupture qu'ils 
opèrent avec le monde ancien, avec le temps du monde d'autrefois, avec les chaînes de la tradition, avec le cours inéluctable de l'histoire des siècles passés sous la tyrannie, la misère, l'injustice. C'est ce rejet du temps passé que reproche Edmund Burke à l'un des plus radicaux des révolutionnaires, Thomas Paine en 1790 lors de la publication de ses Rights of Man : comme Thomas Jefferson, ce dernier nie le pouvoir « des morts sur les vivants », et le poids des traditions sur la modernité (Burke, 195).

Franklin (qui meurt d'ailleurs la même année) n'aurait pas dû subir l'ire de Burke car, s'il est loin d'être un conservateur, il ne rejette pas la légitimité du rôle de l'histoire, au moins dans ce qu'elle peut apporter à la polémique dont il raffole: n'a-t-il pas ressuscité, à l'instar de Jefferson, mais avec dérision, la vieille Germanie dont ils ont fait, par ses anciennes institutions supposées libres et démocratiques, l'ancêtre et le modèle des Américains? N'a-t-il pas admiré le mode d'organisation politique des Iroquois, par exemple, dont l'ancienneté sur le sol d'Amérique dépasse de beaucoup celle des Européens?

Homme des Lumières? Franklin se targue de cette qualité, tout en étant renvoyé, par ses détracteurs anciens et nouveaux, à un utilitarisme vulgaire ${ }^{4}$.

De Franklin, qui est souvent perçu comme l'« homme des Lumières » par excellence, on dira qu'il fut plutôt l'un des hommes des Lumières, exemplaire mais éminemment singulier. De maintes façons, il fut un précurseur : si sa vie traversa presque de bout en bout le XVIIIe siècle (1706-1790), il devança de quelques décennies ce qui fut en réalité un « court siècle des Lumières américaines " (Caron et Wulf, 16), lequel ne se manifesta guère qu'à la troisième ou quatrième décennie du "settecento ». Franklin découvre, peut-on dire, les Lumières en fuyant son milieu familial puritain. Il les invente en expérimentant sur lui-même et par l'écrit, dans une forme adaptée au milieu colonial, les valeurs et les principes propres aux Philosophes cosmopolites éclairés; parallèlement, il exalte les sociétés du Nouveau continent, dont il prédit à tort qu'il sera un jour « le siège de l'empire britannique », parce que là résident les conditions et les valeurs qui feront le succès de ce qu'il appelle, lui comme tant d'autres, "a rising people ».

16 Enfin, parmi tous ses avatars ${ }^{5}$, le Pauvre Richard est l'auteur de l'almanach du même nom. L'almanach, premier livre publié aux colonies anglaises d'Amérique, est, depuis l'Antiquité, l'un des lieux où le temps se dévoile sous toutes ses faces : c'est le temps des astres, celui de la terre et de la culture, celui des humeurs du corps et de l'âme, le temps de la cueillette des plantes médicinales; sans compter les proverbes et les maximes qui dictent au lecteur la morale et les traditions qui relient les générations anciennes et nouvelles. L'almanach du "Pauvre Richard", comme, dans une moindre mesure, les journaux que rédigea Franklin, qu'il imprima et diffusa, comme aussi ses charges successives de maitre des postes de Philadelphie puis de toutes les colonies, sont emblématiques de son rapport au temps.

Naturellement, son " Autobiographie ${ }^{6}$ » s'inscrit, par définition, dans le cours de sa vie, mais, outre le contenu de cet ouvrage, sa forme joue avec les différents temps qui sont ceux d'une écriture fragmentée par quelques décennies d'interruption, d'une distance temporelle avec les matériaux d'un journal et de notes fugaces d'un temps envolé, et enfin et surtout, elle contient en réduction les réflexions de l'auteur sur les divers aspects du temps qui l'occupe et ses recommandations à sa postérité, dans un temps qu'il lui arrive de suggérer comme par inadvertance, songeant à ses œuvres et à son âme, éternel. 


\section{Le temps de la vie}

On peut aisément lui appliquer cette phrase qu'écrivait sur lui-même Jean-Pierre Vernant au début de son beau livre d'égo-histoire : «Plusieurs couches sédimentaires distinctes [...], chacune avec son propre mode de temporalité, entrent en résonance " (Vernant, 11) dans la personnalité et la vie de Franklin. d'un plus jeune fils qui fut lui-même le plus jeune du plus jeune (Autobiography, 11). Depuis sa jeunesse, il vise à une grande longévité et, de façon surprenante, malgré sa goutte qu'il soigne si mal', et l'époque qui ne voit guère d'octogénaires, il y atteint. Il mourra à 84 ans, comme d'autres natifs de Nouvelle-Angleterre dont il a pris soin de relever la chronologie sur leurs tombes ${ }^{8}$. Ses propres parents sont morts respectivement à 89 et 85 ans, "sans avoir connu aucune maladie" ayant vécu modestement et vertueusement, ayant consacré leur vie à leur « vocation » et ayant été de ce fait récompensés par la Providence. Dans cette longévité en réalité moins fréquente parmi ses contemporains qu'il ne l'affirme, Franklin trouve la preuve que le climat, la diète et les mœurs des colonies anglaises d'Amérique sont bien plus sains que ceux de la Grande-Bretagne9. Dans son propre cas, il tira fierté d'avoir su, en plus de fortifier son corps par la natation par exemple, étirer en quelque sorte le temps de la vie, non seulement dans le nombre des années, mais dans l'épaisseur qu'il donna à chacune.

Son rapport physique au temps se caractérise par un penchant qu'on pourrait dire vitaliste ou eudémoniste. Il jouit pleinement de chaque minute consacrée au plaisir des sens : sans être un dilettante raffiné comme Jefferson, on connaît son goût pour le vin, pour les femmes, mais aussi une aptitude au bonheur dans tout ce qu'il fait physiquement et intellectuellement. Pour son confort, pourrait-on dire, officieusement bigame, il fait même fi du temps au point de ne pas attendre le décès de son épouse de Philadelphie pour s'établir dans un deuxième foyer à Londres, puis à Passy. Il prend tant de plaisir à vivre qu'il apprécie le passage de chacun des stades depuis l'enfance jusqu'au temps de la vieillesse et que, transposant son plaisir en maximes, il le généralise : "At twenty years of age/ the will reigns; at thirty, the wit; and at forty, the judgment $»^{10}$. Puis vient la sagesse du vieil homme, dont il se fait une posture, si réussie qu'elle le dépassera pour devenir une icône grâce notamment au Poor Richard's Almanach. Commençant son autobiographie par un retour sur sa vie passée, il s'en trouve satisfait au point qu'il «ne verrai[t] aucune objection à [la] recommencer [...] depuis le début "(Autobiography, 1). Mais, puisque la "répétition » de la vie n'est pas possible, il justifie son récit par le recours au souvenir (recollection) : de sorte que le passé revient à la mémoire et s'incarne au présent, dans l'écrit.

21 Plutôt que de faire table rase du passé de sorte à faire valoir sa réussite personnelle, Franklin trace, grâce au fil renoué avec le passé ancestral, une évolution constante vers sa postérité. Il commence en effet par sa généalogie ${ }^{11}$ dont le récit d'un passé hypothétique remontant au XVIe siècle montrerait que les Franklin tenaient leur nom d'un titre fondé sur de prospères francs-alleux, ce qui en faisait des notables locaux. Dès ces temps anciens, les Franklin rejoignirent le camp des réformés, offrant à Benjamin le premier exemple d'une résistance à la corruption et à l'oppression papistes 
et du choix des valeurs morales de rectitude tant en matière religieuse que civile. Benjamin a donc tout lieu de tirer vanité (comme il avoue lui-même qu'il en est coutumier) d'un très lointain passé anglais, dont le cours fut interrompu et modifié lorsque ses parents émigrèrent en Nouvelle Angleterre où il naquit.

Il est cependant une échéance qui échappe à son pouvoir ; c'est la principale raison qui le conduit, à la veille de la mort, à réitérer dans une lettre à Ezra Stiles, le président de Yale, sa foi déiste plutôt que chrétienne, mais attachée à la récompense de ses vertus dans l'éternité. "Il est impossible, argumente-t-il d'ailleurs, que si Dieu a fait toute chose sur terre, éternelle, il n'ait pas pensé à donner à l'homme aussi l'immortalité ${ }^{12}$.

Franklin est un matérialiste avant tout. L'immortalité, c'est surtout le souvenir que l'on laisse après soi si on y a veillé de son vivant : «If you would not be forgotten as soon as you are dead, either write something worth reading or do things worth writing $»^{13}$. La réussite sociale recherchée durant sa vie, Franklin souhaite la prolonger de manière posthume par ses écrits. Curieusement, toutefois, sans doute à cause de ses qualités d'abord pragmatiques, son bon sens et son souci primordial de l'utilité des actions comme des écrits, il n'a pas laissé de grand œuvre littéraire ni philosophique. En revanche, l'acuité de son humour, de ses traits d'esprit et de ses jeux de mots, son imagination inépuisable comme son goût pour les fables, mais aussi son intérêt pour la spéculation philosophique font de ses Almanachs des ouvrages d'une valeur non négligeable et bien supérieure aux autres almanachs qui se publiaient dans chaque province. Son érudition, le ton à la fois sentencieux et narquois de ses almanachs, la rudesse de beaucoup de ses réflexions et réactions politiques ne le cèdent en rien à la finesse de sa pensée et à son adéquation aux nécessités du temps. Si lui-même ne produit pas de chef d'œuvre littéraire, il est admiré comme le découvreur de l'électricité (rappelons qu'il la rapporte à la foudre: encore une manifestation du «temps » produit par les éléments). Ce grand pas qu'il fait faire à la science s'inscrit dans les recherches des savants de son époque qui le reconnaissent comme un des leurs. Mais il est aussi l'auteur du premier paratonnerre, proposant au monde de son époque l'un de ces objets que l'on n'oubliera pas car dessinant déjà la technologie de l'avenir.

sorte que la gloire qu'il réussit à atteindre de son vivant le suivra, comme il l'espérait, dans sa postérité. On est là au cœur du paradoxe que l'homme portait en lui et qu'il inscrivit dans sa vie et ses écrits : homme pratique avant tout qui savait, par l'invention d'objets utiles, remédier par exemple à l'affaiblissement de la vision (les verres à double foyer) ou prolonger sa main d'un long manche à crochet pour la hisser jusqu'aux étagères les plus hautes de sa librairie. C'est encore lui qui eut l'idée d'aménager les cheminées et les poêles de sorte à répandre l'air chaud dans les intérieurs.

Inutile de revenir ici sur les accomplissements de Franklin imprimeur, journaliste, homme de sciences, inventeur, diplomate, homme d'Etat ni d'énumérer la longue liste de ses écrits qui font plus de trente volumes de l'édition de ses Papers. Inutile de faire ici son éloge funèbre, sauf pour rappeler la qualité du citoyen qui ne se contenta pas de rechercher la gloire, mais se mit au service de ses concitoyens de Philadelphie dont il fit une communauté urbaine moderne par l'aménagement des rues, la compagnie de pompiers, la bibliothèque... Benjamin Franklin a fait pénétrer la modernité des Lumières à Philadelphie. Regardant en avant dans un souci didactique ainsi que dans le désir de faire briller son image post-mortem, Franklin adhère donc à la conception du 
temps des Lumières : le temps de la modernité, un temps en mouvement, un «temps dans le temps des hommes ", concrétisé par l'almanach, dans lequel Franklin note, par exemple, les anniversaires de la mort des hommes célèbres, comme Newton, Addison, Locke, Sir Walter Raleigh, etc., un temps généralement linéaire, mais qui peut redevenir circulaire si l'individu ne maitrise pas, par sa vertu, sa destinée : on voit ainsi dans l'Autobiographie comment l'imprimeur Keimer qui ne sait pas gérer son entreprise, se trouve aux prises avec la vieille Roue de la Fortune moyenâgeuse qui s'empare du destin du fautif, faisant de lui l'employé de celui qu'il dominait autrefois (Autobiography, 55).

De fait, à la différence des nombreux récits de vie qui paraissent alors, l'Autobiographie de Franklin n'est pas exactement linéaire. Certes, parlant de lui-même, et voulant s'en justifier, il est aisé pour ce citoyen modèle et volontariste, de tracer rationnellement la chaîne temporelle de l'avant et de l'après. Tout en vivant intensément le moment présent, il laisse s'écouler en un flot continu la succession des générations depuis ses lointains ancêtres, jusqu'aux événements de sa propre vie et au souci qu'il entretient d'éduquer ses lecteurs par son exemple. Passé, présent, avenir s'alignent ainsi dès la présentation de son Autobiographie, tout en se chevauchant parfois de manière redondante : dès la première page, après avoir rappelé à son fils l'enquête qu'ils firent en Angleterre trois décennies auparavant à la recherche des traces de ses propres ancêtres (négligeant le fait que ce sont aussi ceux de son fils), il se place au centre du récit et promet à son fils des plaisirs comparables à la lecture des « anecdotes » qui ont marqué sa vie. En outre, les moyens par lesquels il a "émergé hors de la pauvreté et de l'obscurité dans lesquelles [il est] né jusqu'à atteindre l'état d'affluence et de haute réputation qui lui procurent une telle félicité dans [son] grand âge " fourniront aux générations à venir un exemple utile et même nécessaire ${ }^{14}$.

Enfin, Franklin est si confiant en lui-même qu'il peut avouer dans ses vieux jours : « For having lived long, I have experienced many instances of being obliged, by better information or fuller consideration, to change opinions, even on important subjects, which I once thought right but found to be otherwise $»^{15}$. Hormis les imprudences de jeunesse à Londres, l'une de ses principales erreurs fut peut-être celle de rester attaché trop longtemps à la permanence d'un empire britannique amélioré par quelques réformes salutaires auquel il croyait encore dans les années 1760. Plus tard, devenu l'un des dirigeants révolutionnaires, la sagesse a consisté à renoncer dans son vieil âge au conservatisme pour adopter des vues si radicales que, délégué et doyen respecté de la Convention constitutionnelle en 1787, ses options démocratiques (comme le monocamérisme) restèrent inaudibles aux oreilles conservatrices des signataires de la Constitution. Mais c'est grâce à l'astuce du vieux sage que les délégués purent sortir de l'impasse dans laquelle ils se trouvèrent lorsque, la Constitution enfin rédigée en septembre 1787, ils faillirent ne pas avoir l'unanimité des signatures des Etats, sauf à faire signer les délégués eux-mêmes sans prêter attention à ceux qui s'étaient dérobés.

\section{Le temps utile : « Le temps, c'est de l'argent »}

Le soir du 17 avril 1790 se clôt la durée de vie de Benjamin Franklin. L'œuvre qu'il laisse est innombrable et une grande partie a franchi le temps qui nous sépare de cette époque. L'un de ses plus importants accomplissements est sa pensée économique qui, sans avoir donné l'occasion d'un ouvrage théorique, forme un ensemble à travers tous 
les écrits qui parlent de divers aspects de l'économie du XVIIIe siècle - agriculture, commerce, artisanat, finances, richesse et pauvreté. A cet égard, la relecture des deux chapitres qu'y a consacré Christian Lerat dans son ouvrage de 1992 (Lerat) fournira une substance plus approfondie que l'approche que j'en ferai ici en parlant du rôle que joue « le temps » dans la pensée de Franklin sur l'argent, le travail et la richesse.

Dès son enfance, raconte Franklin en 1779, dans une "bagatelle ", "The Whistle» ${ }^{16}$, destinée à l'une des dames qu'il courtise à Paris, il a découvert à ses dépens la valeur de l'argent en achetant trop cher un sifflet, objet fruste qui a été fabriqué en quelques minutes, et dont le travail fourni ne valait pas de loin le prix qu'il l'avait payé. Dès lors, il a consacré une grande partie de son énergie vitale à produire de l'argent par le travail, par l'économie, par l'organisation judicieuse de son temps.

En 1748, à quarante-deux ans, il s'estime assez fortuné grâce à son propre travail pour prendre sa retraite d'imprimeur et la consacrer à l'étude et au bien public. C'est en connaissance de cause qu'il peut alors se permettre de donner les conseils d'un « old tradesman " à un débutant ${ }^{17}$ et le premier d'entre ses " commandements " comme dit Christian Lerat, aura une réputation universelle : «Remember that time is money ». Si, comme on l'a vu, «time is the stuff life is made of», et si «time is money, » on peut alors s'amuser à dire que pour Franklin : « l'argent, c'est la vie », ou vice-versa.

31 Tout d'abord, virtuose dans la gestion de son temps quotidien, il se sent apte à donner des leçons en cette matière : « He that rises late must trot all day.» ${ }^{18}$. La bonne heure du lever est cinq heures du matin; celle du coucher dix heures du soir - ce qui aide à économiser les chandelles, mais aussi à rendre la journée profitable et rationnelle: Franklin adore faire des plans, le plus célèbre étant le catalogue des Vertus qu'il se propose d'acquérir. Moins connu, le "Scheme of Order $»^{19}$, est une "charpente temporelle » faussement naïve qui trace le plan d'une journée idéale, depuis le matin où l'on doit se demander : «que vais-je faire de bien aujourd'hui? » jusqu'à la question du soir: "qu'ai-je fait de bon aujourd'hui?». Pour dans l'intervalle, partager sa journée entre le travail (six heures) et la lecture, la musique, les divertissements, les repas: un emploi du temps qu'il a fait l'effort de suivre étant tout jeune quand, apprenti imprimeur, il laissait ses compagnons aller boire et manger à la taverne pendant qu'il restait à l'atelier à lire et boire de l'eau, économisant ainsi le temps et l'argent, mais qu'il reconnaît mal adapté à l'entrepreneur, plus sollicité (Autobiography, 28).

L'important est de maîtriser aussi bien ses loisirs que son temps de travail, le but étant d'augmenter ses moyens matériels et, si possible, de faire fortune. "Rappelle-toi que l'argent est doté de puissance génitale et de fécondité et que les rejetons peuvent engendrer à leur tour et ainsi de suite", écrit-il dans son "Advice to a young Tradesman » de 1748. Et faisant le compte et le décompte de ce que le paresseux gaspille à jouer et de ce qu'il perd en ne travaillant pas, il calcule que le prodigue a ainsi «jeté cinq shillings à l'eau » alors qu'il aurait pu en gagner dix en travaillant toute la journée. Mais même si, comme le souligne Christian Lerat, la pensée économique de Franklin sur le travail, fondement de la valeur de l'argent, est allée jusqu'à inspirer Karl Marx, il ne s'agit pas pour notre personnage de céder à la tentation de diminuer le temps de loisir pour augmenter indéfiniment le temps du travail. Franklin ne sacrifiait pas la qualité à la quantité. Il donnait même au temps du loisir une importance aussi grande qu'à celui du travail, à condition qu'il soit fructueux : «Leisure is the time for doing something useful. This leisure the diligent person will obtain/the lazy one 
never $\aleph^{20}$. Benjamin Franklin conçoit une société où le temps du travail se complète d'un temps de loisir qui permet l'édification d'une culture de la civilisation, des BeauxArts et de la science. Franklin déploie, dans de nombreux pamphlets, l'image des progrès qu'ont connus et que connaîtront encore les colonies du Nouveau Monde: d'une société fruste et matérialiste, établie dans l'urgence par les premiers immigrants, les colonies anglaises d'Amérique évoluent vers une société raffinée qui fait sa place aux « virtuoses et aux génies » à l'égal des sociétés européennes. Bien qu'éloignées du Vieux monde et voisines des "sauvages", elles réussissent à égaler les sociétés européennes et à prendre place dans l'histoire mondiale de la civilisation. C'est surtout sous cet aspect du progrès que les Lumières sont parvenues en Amérique.

Si le progrès est l'un des grands principes qui dominent au XVIIIe siècle, c'est que l'idée en est permise par une nouvelle conception du temps. L'ouvrage de Condorcet, Esquisse d'un tableau historique des progrès de l'esprit humain (1795), histoire prospective d'une marche sans fin de l'humanité vers la perfection, n'est que le plus célèbre d'innombrables articles, fascicules et ouvrages qui placent tous leurs espoirs d'avenir dans une amélioration continue et sans limites du genre humain. Les Lumières s'emparent de cette conception d'un avenir de gloire et de paix, qui efface de la mémoire le passé et son obscurité, et oblitère même le présent comme temps autonome, n'y voyant que le premier pas de la marche vers des lendemains heureux. 
39 Adepte $\mathrm{du}$ principe de progrès et nationaliste, Franklin, comme d'autres commentateurs par la suite, voit dans l'Amérique l'endroit de la planète qui rassemble le mieux les conditions où s'exercera cet esprit des Lumières fait d'espoir dans l'avenir, de confiance dans l'homme et de liberté naturelle. La rapide croissance de la démographie des colons ${ }^{23}$, leur environnement physique et les qualités dont ils sont parés concourent à en faire le peuple peut-être le plus éclairé au monde ${ }^{24}$.

Pour certains des contemporains de Franklin, cette marche vers l'avenir, conçue hors de toute visée eschatologique chrétienne, se déroule tout entière dans le monde icibas : par le progrès technique et scientifique s'annonce une civilisation dans laquelle l'homme, déchargé des labeurs les plus pénibles, assaini par la médecine, épargné du besoin, sera par là même délesté de son poids de vices, de malheurs, d'agressivité. Outre la paix qu'apportera la prospérité générale, l'humanité jouira du bonheur et s'adonnera, dans des régimes politiques justes et libres, à la jouissance de la liberté.

41 Il est vrai que l'on ne retrouve pas cette utopie chez le pragmatique avisé qu'est Franklin, dont l'optimisme reste mesuré, bien qu'il soit aussi attaché à sa "nouvelle patrie » que ses amis nationalistes. S'il dessine pour les États-Unis naissants un destin impérial, il prône pour les individus et à l'intérieur même de la société, une «happy mediocrity » que, en démocrate qui s'ignore presque, il fait reposer sur une population en majorité agraire - ces petits cultivateurs qui sont le « sel de la terre » selon Jefferson -, et sur l'opposition aux sociétés d'ordre nobiliaire ou d'oligarchie qu'il renie.

42 Franklin ne s'intéresse guère à l'histoire. Parmi ses lectures, peu d'historiens, sauf Plutarque dont les Vies forment une histoire plutôt tendancieuse, et, dans ses réflexions, pas de références à l'histoire, sauf lorsqu'il évoque ses ancêtres qui avaient participé au mouvement de réforme et qui furent parmi les dissenters en Angleterre: autant de preuves qu'il est en quelque sorte prédestiné à la liberté et aux qualités qui font le terreau des colonies. Comme ses contemporains, l'enseignement de l'histoire lui semble nécessaire à une bonne éducation, surtout pour les exemples de vertu qu'elle offre ${ }^{25}$. En revanche, dans «A Proposal for Promoting Useful Knowledge among the British Plantations in America $»^{26}$ de 1743, il applique aux colonies américaines la théorie des stades successifs traversés par les sociétés humaines, partageant avec son temps les grands principes d'une philosophie de l'histoire universelle. D'autre part, il abonde, bien que dans un style parodique, dans le sens de Jefferson et d'Adams dont la référence, d'une historicité douteuse, à un passé saxon ${ }^{27}$ fait argument dans le conflit impérial.

\section{Conclusion}

Maîtrise du temps de la vie, invention du temps économique, foi dans la capacité des hommes à s'éduquer et à améliorer leur bien-être et à connaître le monde : tel a voulu être ce génial bourgeois, dans un optimisme tempéré par une prudente méfiance dans les relations d'intérêt ${ }^{28}$. Par son exemple et ses exhortations, il a préparé son pays à l'aventure du capitalisme dans le siècle qui le suivra. Par son audace, sa singularité, son intelligence, il a su rejoindre et propulser l'esprit de liberté qui a régné dans les Lumières atlantiques. 


\section{Sources primaires}

BURKE, Edmund. Reflections on the Revolution in France, (1790), ed. By Conor Cruise O'Brien.

London : Penguin Books, 1968.

FRANKLIN, Benjamin. Benjamin Franklin's Autobiography, ed. by Leo Lemay and P.M.Zall. New York : Norton, 1986.

FRANKLIN, Benjamin. The Papers of Benjamin Franklin, Sponsored by The American Philosophical Society and Yale University, Digital Edition by The Packard Humanities Institute, 2002-2008. http://www.franklinpapers.org/ consulté pour la dernière fois le 4 février 2009.

FRANKLIN, Benjamin. Writings, Leo Lemay ed. The Library of America. New York : Viking, 1987.

\section{Sources secondaires}

BRAUDY, Leo. The Frenzy of Renown. Fame and its History. New York : Random House, Vintage, 1990 CARON, Nathalie, et Naomi WULF. «Introduction : Les lumières américaines dans l'historiographie contemporaine aux Etats-Unis : ambivalences et réticences», Revue française d'études américaines, Les lumières américaines, 92, 2002 (2), pp. 3-21.

CORBIN, Alain. L'avènement des loisirs, éd. Paris : Champs Flammarion, 2008.

GREENE, Jack P. The Intellectual Construction of America : Exceptionalism and Identity from 1492 to 1800. Chapel Hill, N.C. : University Press of North Carolina, 1993.

GROETHUYSEN, Bernard. Philosophie et histoire, éd. par Bernard Dandois. Paris : Albin Michel, 1995.

HUGHES, Gérard. in "Benjamin Franklin, des Lumières à nos jours", Etudes anglaises, (95), 1992, p. 19.

LERAT, Christian. Benjamin Franklin : Quand l'Amérique s'émancipait. Bordeaux : Presses de l'université de Bordeaux, 1992.

MARIENSTRAS, Elise. Les mythes fondateurs de la nation américaine. (1976), Paris : Editions Complexe, 1992.

MARIENSTRAS, Elise. «'Etc' , ou, Du bon usage de la démographie chez Benjamin Franklin », in L'idée américaine au XVIIIe siècle, collection Frontières, Paris : Presses de l'université ParisSorbonne, 1992.

PAGDEN, Anthony. European Encounters with the New World : From Renaissance to Romanticism, New Haven, Yale University Press, 1993.

PIGEARD DE GUIBERT, Guillaume, Kate E. TUNSTALL et Jean SALEM éds. Qu'est-ce que les Lumières?, Oxford : Voltaire Foundation, Studies on Voltaire and the Eighteenth Century, 2006.

VERNANT, Jean-Pierre. La Traversée des Frontières, entre mythe et politique II. Paris : La Librairie du XXIe siècle, éd. du Seuil, 2004. 


\section{NOTES}

1. Poor Richard Improved, in Benjamin Franklin, Writings, Leo Lemay ed., The Library of America, 1987, 1296.

2. Si Franklin ne s'intéresse guère à la survie de l'âme à laquelle il dit parfois croire, il ne dédaigne pas la renommée qu'il souhaite aussi bien posthume que présente. Voir Leo Braudy, The Frenzy of Renown. Fame and its History, New York, Random House, Vintage, 1990, 368-69.

3. In « Silence Do Good, $\mathrm{N}^{\circ} 10$ », Benjamin Franklin, Writings, op.cit., 27.

4. Voir in Benjamin Franklin's Autobiography, ed. by Leo Lemay and P.M. Zall, New York, Norton, 1986, "Criticism ", 244-46, l'article de John Adams publié dans le Boston Patriot du 15mai 1811, plein de jalousie et de fiel contre un Franklin accusé de vanité, d'hypocrisie et d'imposture. Voir aussi ibid., 289-99, D.H. Lawrence, "Benjamin Franklin», extrait des Studies in Classic American Literature (London: M. Secker, 1924).

5. Voir dans ce même numéro, l'article de Michael Zuckermann.

6. On a utilisé ici l'édition annotée par Leo Lemay et P.M. Zall, Benjamin Franklin's Autobiography, op.cit.

7. «Dialogue Between The Gout and Mr Franklin », in Writings, op.cit., 943-50.

8. "Observations Concerning the Increase of Mankind, Peopling of Countries, etc. », Writings, 367-74.

9. Voir « American Longevity », 1767, in Papers, vol.14, 337.

10. Poor Richard's Almanac, June (1741) Writings, op.cit., 1219.

11. Voir aussi « Genealogical Chart of the Franklin Family »,in Papers, vol. 14, 120.

12. « To Ezra Stiles », Writings, op.cit., 1179.

13. Poor Richard's Almanach, 1738, Papers, vol. II, 190.

14. in Papers, III, 306-307.

15. «Speech in the Convention at the Conclusion of its Deliberations », in Writings, op.cit., 1139.

16. In Writings, op.cit., 931-33.

17. « Advice to a young Tradesman », 1748, in Papers, III, 306-307.

18. « Poor Richard Improved », 1758, in Writings, op.cit., 1296.

19. Autobiography, op.cit., 78-79. Nous empruntons l'heureuse expression de «charpente temporelle » à Alain Corbin dans L'avènement des loisirs, éd. Champs Flammarion, 2008.

20. «Poor Richard Improved, 1758 », Writings, op.cit., 1299.

21. Max Weber, The Protestant Ethic and the Spirit of Capitalism, cit. in Autobiography, op.cit., 280-284.

22. «Necessary Hints To Those Who Would Be Rich» (1736), in Jared Sparks, ed., The Works of Benjamin Franklin: Containing Several Political and Historical Tracts ... Letters, Official and Private ..., (Boston, 1940).

23. "Observations concerning the Increase of Mankind, Peopling of Countries, etc », Benjamin Franklin, Writings, op.cit., 367-74. Voir Elise Marienstras, «Etc., ou, Du bon usage de la démographie chez Benjamin Franklin » in L'idée américaine au XVIIIe siècle, collection Frontières, Presses de l'Université Paris-Sorbonne, 1992.

24. «To David Hartley», Sat, Sep 6, 1783, Writings, op.cit. unpub.

25. «Proposals Relating to the Education of Youth in Pensilvania (sic)», in Writings, op.cit., 336-340.

26. "A Proposal for Promoting UsefulKnowledge among the British Plantations in America », 1743, in Writings, op.cit. 295-97.Voir aussi Jack P. Greene,The Intellectual Construction of America: Exceptionalism and Identity from 1492 to 1800 , Chapel Hill, The University of North Carolina Press, 1993, 117-119.

27. «Dialogue Between Britain, France, Spain, Holland, Saxony, and America », in Writings, op.cit., 725-728. 
28. Voir Gérard Hughes, « Benjamin Franklin : le paradoxe » in "Benjamin Franklin, des Lumières à nos jours", G. Hugues et D. Royot eds. Études anglaises (95), 1991, 19.

\section{RÉSUMÉS}

La renommée multiséculaire de Benjamin Franklin tient surtout à sa qualité d'homme des Lumières. Cet article s'intéresse à l'extrême complexité de sa pensée sur le temps comme concept, comme mode de vie, comme outil pour s'enrichir, et comme vision générale du progrès du genre humain.

The long-lasting fame of Benjamin Franklin rests principally on his characteristics as a scientist and a philosopher of the Enlightenment. This article raises the question of Franklin's conception of time and examines his numerous uses of «time » as a concept, as a way of living, as a means to obtain wealth, as a general vision of the progress of mankind.

\section{INDEX}

Mots-clés : Bonheur, imprimerie, loisir, progrès, richesse, sciences

Keywords : Happiness, leisure, printing, progress., sciences, wealth

\section{AUTEUR}

\section{ELISE MARIENSTRAS}

Université Paris-Diderot

Professeur émérite

elise.marienstras@free.fr

Elise Marienstras, professeur émérite à l'Université Paris-Diderot, a enseigné l'histoire des ÉtatsUnis. Son intérêt se porte depuis longtemps sur les problèmes posés par la formation nationale à la fin du XVIIIe siècle dans ce pays de colonisation de peuplement qui s'est bâti sur le face-à-face inégal entre les cultures conquérantes et résistantes. Elle a publié notamment:Les mythes fondateurs de la nation américaine, (1976) Complexe, 1992.

La résistance indienne aux États-Unis, éd. Gallimard, 1980.

Nous, le Peuple. Les origines du nationalisme américain, éd. Gallimard, 1988.

1890. Wounded Knee. L'Amérique au tournant du siècle, Complexe, 1992. 